\title{
Very high-energy observations of the two high-frequency peaked BL Lac objects 1ES $1218+304$ and $\mathrm{H} 1426+428$
}

\author{
C. Mueller ${ }^{\mathrm{a} 1}$, N. Akhter ${ }^{\mathrm{b}}$, J. Ball ${ }^{\mathrm{c}}$, D.A. Bramel ${ }^{\mathrm{b} 3}$, J. Carson ${ }^{\mathrm{c}}$, C.E. Covault ${ }^{\mathrm{d}}$, D. \\ Driscoll $^{\mathrm{d} 5}$, P. Fortin ${ }^{\mathrm{b} 6}$, D.M. Gingrich ${ }^{\mathrm{e}}$, D.S. Hanna ${ }^{\mathrm{a}}$, A. Jarvis ${ }^{\mathrm{c} 7}$, J. Kildea ${ }^{\mathrm{a} 8}$, T.

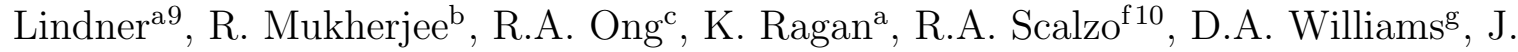 \\ Zweerink $^{\mathrm{c}}$
}

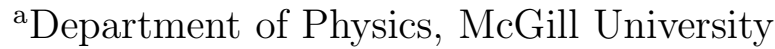

Montreal, QC H3A 2T8, Canada

${ }^{\mathrm{b}}$ Department of Physics and Astronomy, Barnard College, Columbia University

New York, NY 10027, USA

${ }^{\mathrm{c}}$ Department of Physics and Astronomy, University of California, Los Angeles Los Angeles, CA 90095, USA

dDepartment of Physics, Case Western Reserve University Cleveland, $\mathrm{OH}$ 44106, USA

${ }^{\mathrm{e}}$ Centre for Particle Physics, Department of Physics, University of Alberta Edmonton, AB T6G 2G7, and TRIUMF, Vancouver, BC V6T 2A3, Canada

${ }^{\mathrm{f}}$ Department of Physics, University of Chicago Chicago, Il 60637, USA

gSanta Cruz Institute for Particle Physics, University of California, Santa Cruz Santa Cruz, CA 95064, USA

\footnotetext{
${ }^{1}$ present address: Sander Geophysics Limited, Ottawa, ON K1V 1C1 ${ }^{2}$ present address: Gemini North, Hilo, HI 96720, USA ${ }^{3}$ present address: Interactive Brokers, Greenwich, CT 06830, USA

${ }^{4}$ present address: The Claremont Colleges, Joint Sciences Department, Claremont, CA 91711-5916, USA ${ }^{5}$ present address: Kent State University, Ashtabula, OH 44004, USA

${ }^{6}$ present address: Laboratoire Leprince-Ringuet, École Polytechnique, CNRS/IN2P3, Palaiseau, France ${ }^{7}$ present address: Disney Interactive Media Group, North Hollywood, CA 91601, USA

${ }^{8}$ present address: McGill University, Medical Physics Unit, Montreal, QC H3G 1A4

${ }^{9}$ present address: Department of Physics and Astronomy, University of British Columbia, Vancouver, BC V6T 1Z1, Canada

${ }^{10}$ present address: Department of Physics, Yale University, New Haven, CT 06520, USA
} 


\begin{abstract}
We present results of very-high-energy gamma-ray observations $\left(E_{\gamma}>160 \mathrm{GeV}\right)$ of two high-frequency-peaked BL Lac (HBL) objects, 1ES 1218+304 and H 1426+428, with the Solar Tower Atmospheric Cherenkov Effect Experiment (STACEE). Both sources are very-high-energy gamma-ray emitters above $100 \mathrm{GeV}$, detected using ground-based Cherenkov telescopes. STACEE observations of 1ES 1218+304 and H 1426+428 did not produce detections; we present 99\% CL flux upper limits for both sources, assuming spectral indices measured mostly at higher energies.
\end{abstract}

\title{
1. Introduction
}

Nearly thirty active galaxies of the "blazar" class have been detected as very-high-energy gamma-ray sources using ground-based atmospheric Cherenkov telescopes (ACTs) (e.g. see review [1]). Blazars are compact, highly variable extragalactic objects, characterized by non-thermal continuum emission that extends from radio to very-high-energy gamma rays. These sources are broadly classified into two groups: BL Lacertae (BL Lac) objects and flat-spectrum radio quasars (FSRQs). BL Lac objects generally have smooth, featureless continuum spectra, with emission lines that are weak or absent. The spectral energy distributions (SEDs) of these sources typically have two broad peaks, one at low energies (radio to X-ray) and the other at higher energies (keV to $\mathrm{TeV}$ ). Blazars are thought to be highly beamed sources, with relativistic jets oriented close to the line of sight [ 2]. In the current blazar paradigm, the low energy peak in the blazar SED is explained as synchrotron emission from high-energy electrons in the jet, while the high-energy emission is due to relativistic charged particles in the blazar jet. In leptonic models, high-energy gamma rays are produced by inverse Compton (IC) scattering of ambient low energy photons by relativistic electrons. In an alternate scenario, hadronic models explain the gamma-ray energy emission as due to neutral pions produced by energetic protons (e.g. see [3] and [4] for reviews).

Blazars are broadly categorized into sub-groups based on the synchrotron peak frequencies and the relative power in the low and high-energy peaks of their SEDs [ [5]. Of the blazars belonging to the BL Lac class, low-frequency-peaked blazars (LBLs) have this peak in the radio or optical band while for high-frequency-peaked blazars (HBLs), it is in the X-ray band. Historically, the majority of the EGRET-detected blazars belong to the FSRQ class, with the synchrotron peak in the radio-optical band [ 6]. In its first 5.5 months of observations, Fermi detected $21 \mathrm{TeV}$-selected blazars of which 13 are HBLs [ 7]. Since then, the list of Fermi-detected blazars has grown and the first catalog of active galactic nuclei (AGN) detected using the Fermi Large Area Telescope includes 671 gamma-ray sources located at high galactic latitudes that are associated statistically with AGNs [8]. All but a handful of TeV blazars detected to date belong to the HBL category. The exceptions include BL Lacertae, detected using the MAGIC telescope [9], and W Comae, 3C 66A, and PKS1424+240, recently detected in TeV gamma rays by the VERITAS ([10], [[11],[[12]) collaboration. STACEE carried out an extensive observing campaign on two LBLs, 3C 66A and OJ 287, but did not detect any significant gamma-ray emission from either source ([13], [14]). The only blazar detected using STACEE was the HBL Mrk 421 in observations carried out in 2001 [15] and 2004, when STACEE made the first 
measurement of the differential energy spectrum of the source between $130 \mathrm{GeV}$ and 400 $\mathrm{GeV}[16]$.

HBLs have been predicted to be good candidates for TeV gamma-ray emission, based on synchrotron self-Compton (SSC) emission models [17] as well as hadronic models [18. Several of the "extreme" synchrotron BL Lacs [19] have been detected at TeV energies, confirming these predictions. Both H $1426+428$ and 1ES $1218+304$ were predicted to be $\mathrm{TeV}$ gamma-ray emitters, and this was part of the motivation for STACEE to observe these sources. Neither of these sources was detected by EGRET at GeV energies, while both have been detected with Fermi.

1ES $1218+304$ is an X-ray-bright (flux in the $2-10 \mathrm{keV}$ range $\sim 2 \times 10^{-11} \mathrm{erg} \mathrm{cm}^{-2}$ $\left.\mathrm{s}^{-1}[20]\right)$ HBL, and at a redshift of $z=0.182$, it is one of the more distant VHE blazars detected to date. A detection of 1ES 1218+304 was recently reported by both MAGIC [9] and VERITAS [21], at energies $>100 \mathrm{GeV}$, providing further evidence that X-ray-bright HBLs tend to be strong VHE sources. The source is detected with Fermi with no evidence for variability [7]. The MAGIC detection of $\mathrm{TeV}$ emission from this source motivated the observations by STACEE that were carried out in the 2006 and 2007 observing seasons.

$\mathrm{H} 1426+428$ is classified as an "extreme" BL Lac, with its synchrotron peak at an energy greater than $100 \mathrm{keV}$; it has long been predicted to be a TeV emitter. The source was first detected at TeV energies by the Whipple collaboration [22] and later confirmed using other ground-based imaging atmospheric Cherenkov telescopes [ 24, 25]. Like 1ES $1218+304$, its distance $(z=0.129)$ makes it a promising candidate for studying the extragalactic infra-red background radiation. Fermi has detected weak emission from H $1426+428$ at energies less than $20 \mathrm{GeV}$ [ 7]. STACEE carried out observations of H 1426+428 in 2003 and 2004.

The Solar Tower Atmospheric Cherenkov Effect Experiment (STACEE) was a groundbased experiment sensitive to gamma rays above $100 \mathrm{GeV}$. STACEE operated from 2001 until its de-commissioning in the summer of 2007 [26]. STACEE observed several active galaxies of the blazar class with the aim of understanding particle acceleration and emission mechanisms in these sources. With an energy threshold close to $100 \mathrm{GeV}$, STACEE also had the potential to study the effect of the extragalactic background light (EBL) on the spectra of distant blazars. $\S 2$ gives a brief overview of the STACEE instrument. In $\S 3$ we describe the data taking and observing strategy of STACEE, and in $\S 4$ we present results from STACEE observations of 1ES 1218+304 and H 1426+428.

\section{The STACEE Detector}

The STACEE detector used the atmospheric Cherenkov technique, reconstructing veryhigh-energy gamma rays by observing Cherenkov photons resulting from the gamma-ray interactions in the upper atmosphere. The instrument used 64 of the 212 heliostats of the National Solar Thermal Test Facility (NSTTF), near Albuquerque, NM, to direct the Cherenkov light to secondary mirrors, and then onto cameras composed of photomultiplier tubes (PMTs), such that each PMT viewed only a single heliostat. Each heliostat had an area of $37 \mathrm{~m}^{2}$, leading to a large primary mirror area of approximately $2400 \mathrm{~m}^{2}$ and sensitivity to low photon densities (ie, to low gamma-ray energies). The recorded PMT information was used as a measure of the Cherenkov photon impact points on the ground; 
analysis of the time of arrival of the Cherenkov wavefront allowed the reconstruction of the arrival direction and energy of the gamma ray, and it improved the rejection of charged cosmic rays which constituted the main background.

The Cherenkov wavefront is typically only a few nanoseconds in duration, and STACEE used three-level trigger electronics to record Cherenkov events. Each PMT signal was ACcoupled and then split, with one copy going to a rapid (1 GS/s) 8-bit digitizer and one copy going to a discriminator set to detect pulses larger than approximately 5 photoelectrons. The discriminated logic signals were then sent to a custom trigger system [26, 27] which examined the 64 signals in eight clusters of eight channels. Each cluster was considered to trigger if at least five of its constituent channels contained pulses in a 24-nanosecond window, and an event trigger was generated if five of the eight clusters triggered in a 16-nanosecond window. More details of the STACEE detector and operations are given elsewhere [26, 28].

\section{Data Taking and Observing Strategy}

STACEE recorded astrophysical source data in an "ON-OFF" observing mode consisting of a 28-minute ON-source run where the source was tracked at the centre of the field of view, followed by an OFF-source run of the same duration, when a patch of the sky at identical declination but 30 minutes ahead or behind the source in right ascension was observed. The "OFF" run was used to determine the background, based on the facts that a gamma-ray excess is expected only from the source direction, and that the rate of background showers from charged cosmic rays is the same for both runs. The gamma-ray flux was inferred from the difference in count rates between the ON-OFF pair of runs for a particular source observation. Prior to astrophysical source observations, the discriminator threshold was adjusted to eliminate noise triggers resulting from random coincidences of the night sky background. The typical STACEE trigger rate was about $5 \mathrm{~Hz}$. Further details of STACEE nightly operations are given elsewhere [13].

The STACEE data set was analyzed to remove data taken in unfavorable weather conditions or with detector malfunctions (e.g. malfunctioning heliostats, high voltage trips, etc.), in order to eliminate biases in the trigger rates and to increase the sensitivity of the instrument. The STACEE data cleaning criteria are described elsewhere [14]. In addition, field brightness corrections had to be applied to account for the differences between the relative brightness of the ON and OFF fields using a technique called padding [29]1. A crucial step in the STACEE data analysis is the rejection of Cherenkov showers generated by the charged particle background. STACEE used the grid alignment technique for cosmic ray background suppression [ [30, 31, 13]; this technique uses the differences in the distribution of Cherenkov light on the ground for gamma ray and cosmic ray induced showers to provide gamma-hadron separation. Using this analysis method, STACEE reported a detection of the Crab Nebula at a significance of $8.1 \sigma$ in a data set of 21 hours of observation in 2002-2004 [32].

\footnotetext{
${ }^{1}$ Typical field brightness differences led to a difference of trigger rate between ON and OFF fields of a few percent
} 


\section{Results from STACEE Observations of 1ES $1218+304 \&$ H $1426+428$}

\subsection{ES 1218+304}

STACEE observed 1ES 1218+304 during the 2006 and 2007 observing seasons for a total of 70.9 hours (152 ON-OFF pairs) [ 33]. This data set was reduced to 28.3 hours after the standard data quality cuts (referred to above) were applied to the data. The differences in the field brightnesses between the ON and the OFF fields was also taken into account. Table 1 summarizes the livetime available for the 2006 and 2007 data sets. A net ON-source excess of 236 events was seen, compared to a background of 5547 events, corresponding to a statistical significance of $2.3 \sigma$ (calculated using equation (17) in [34]). In the $152 \mathrm{ON}-\mathrm{OFF}$ pairs, there are no individual significances above $4 \sigma$. The $2.3 \sigma$ excess is not statistically significant, and we choose to calculate a flux upper limit for the source, as described below.

In order to interpret the 1ES 1218+304 data and calculate flux upper limits, extensive Monte Carlo simulations of the STACEE detector had to be carried out to determine the effective area of the instrument (see, for example, [14]) as a function of energy and arrival directions for both gamma ray and cosmic ray showers. Figure 1 shows the hourangle-averaged effective area of STACEE for the 1ES 1218+304 observations, weighted according to how much time was spent at each 1ES 1218+304 pointing. The effective area after background rejection cuts is also shown in the figure. As described in [13], the effective area of STACEE below $100 \mathrm{GeV}$ is reduced as a result of the cuts, partly due to the increased pulse-height threshold that is part of the padding process. At energies above $\sim 1 \mathrm{TeV}$, the effective area after cuts is lowered due to the grid alignment cut applied to the data.

The effective area for the 1ES 1218+304 data was used to calculate the detector energy threshold and flux upper limits. We assumed a power-law differential energy spectrum $\frac{d N}{d E} \sim E^{-\Gamma}$ with a spectral index of $\Gamma=3.0$ for $1 \mathrm{ES} 1218+304$, based on the MAGIC [9] and VERITAS [21] measurements. Folding this with the effective area curve, we get an energy threshold $E_{t h}$ of $\sim 155 \pm 28_{\text {sys }} \mathrm{GeV}$ (where the energy threshold is defined by convention as the peak of the resulting detector response function). The systematic uncertainty of $28 \mathrm{GeV}$ arises primarily from uncertainties on the optical characteristics of the experiment (atmospheric attenuation, optical alignment, mirror reflectivities).

We derive a $99 \%$ confidence level (CL) flux upper limit at $155 \mathrm{GeV}$ of $4.7 \times 10^{-10}$ $\mathrm{cm}^{-2} \mathrm{~s}^{-1} \mathrm{TeV}^{-1}$. Figure 2 shows the STACEE upper limit overlaid on the VERITAS and MAGIC spectra. The Fermi point is an extrapolation to $100 \mathrm{GeV}$ from measurements

Table 1

Summary of STACEE data on 1ES 1218+304.

\begin{tabular}{llrrrc}
\hline \hline Year & $\begin{array}{l}\text { Livetime } \\
(\text { hrs })\end{array}$ & $\begin{array}{r}\text { ON-source } \\
\text { Events }\end{array}$ & $\begin{array}{r}\text { OFF-Source } \\
\text { Events }\end{array}$ & $\begin{array}{r}\text { Excess } \\
\text { Events }\end{array}$ & Significance \\
\hline \hline 2006 & 12.0 & 5547 & 5311 & 236 & $2.3 \sigma$ \\
2007 & 16.3 & & & & \\
\hline
\end{tabular}




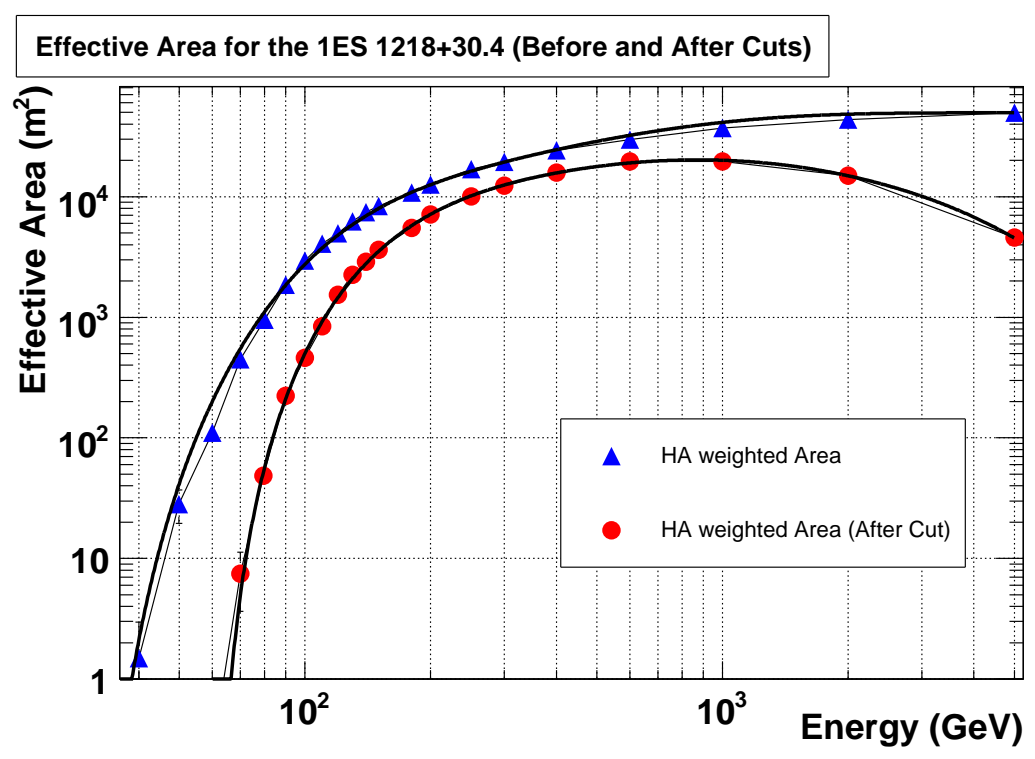

Figure 1. Average effective area of the STACEE detector for the 1ES 1218+304 observations, as a function of gamma-ray energy, after weighting in hour-angle by the time spent at each pointing. The effective area after background rejection cuts is also shown. The solid lines are meant to guide the eye only.

at lower energies, using the Fermi spectral index and no EBL model. The STACEE measurements were carried out at different epochs than the VERITAS, MAGIC, and Fermi measurements. Therefore, the STACEE upper limit is not necessarily in conflict with the two lowest-energy MAGIC data points and the Fermi extrapolation. Blazars are known to be variable sources, and it is possible that the source was not active when STACEE observed it.

\subsection{H $1426+428$}

STACEE observations of the HBL H 1426+428 were performed in 2003 and 2004. The detector was configured differently for the two data sets. In the 2003 data, the heliostats were canted (tilted) to receive light from the position where the air shower created by a primary VHE gamma ray contains a maximum number of charged particle secondaries, at an altitude of approximately $12 \mathrm{~km}$. The canting scheme was changed for the 2004 observations to allow for several heliostats to be aimed directly at the source being observed (i.e. these heliostats were not canted). This change allowed for a better reconstruction of low-energy events and improved background rejection. Because of the different detector sensitivities, the data sets must be treated independently and each one compared to appropriate Monte Carlo simulations. A total of 52.5 hours of data were recorded, with 28.5 hours remaining after standard data quality cuts. Table 2 summarizes the data sets, including the number of ON-source and OFF-source events after selection cuts, and the significance of the ON-source excess. 


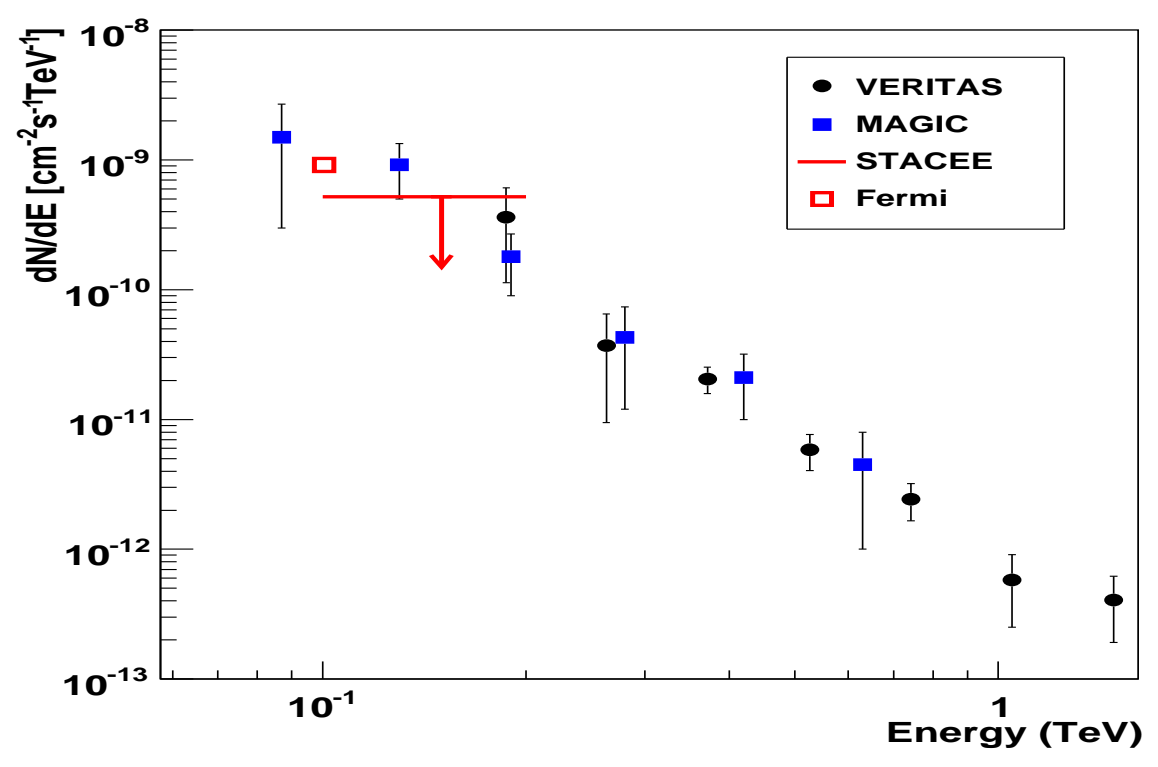

Figure 2. Gamma-ray spectrum of 1ES 1218+304, as measured by VERITAS [21] and MAGIC [9], with the STACEE $99 \%$ flux upper limit at $155 \mathrm{GeV}$. The Fermi point is an extrapolation from lower energy data to $100 \mathrm{GeV}$. Note that the different measurements are not contemporaneous.

Table 2

Summary of STACEE data on H 1426+428.

\begin{tabular}{llrrrc}
\hline \hline Year & $\begin{array}{l}\text { Livetime } \\
(\text { hrs })\end{array}$ & $\begin{array}{r}\text { ON-Source } \\
\text { Events }\end{array}$ & $\begin{array}{r}\text { OFF-source } \\
\text { Events }\end{array}$ & $\begin{array}{r}\text { Excess } \\
\text { Events }\end{array}$ & Significance \\
\hline \hline 2003 & 8.9 & 24480 & 24162 & 318 & $1.6 \sigma$ \\
2004 & 19.7 & 49093 & 49065 & 28 & $0.2 \sigma$ \\
\hline
\end{tabular}




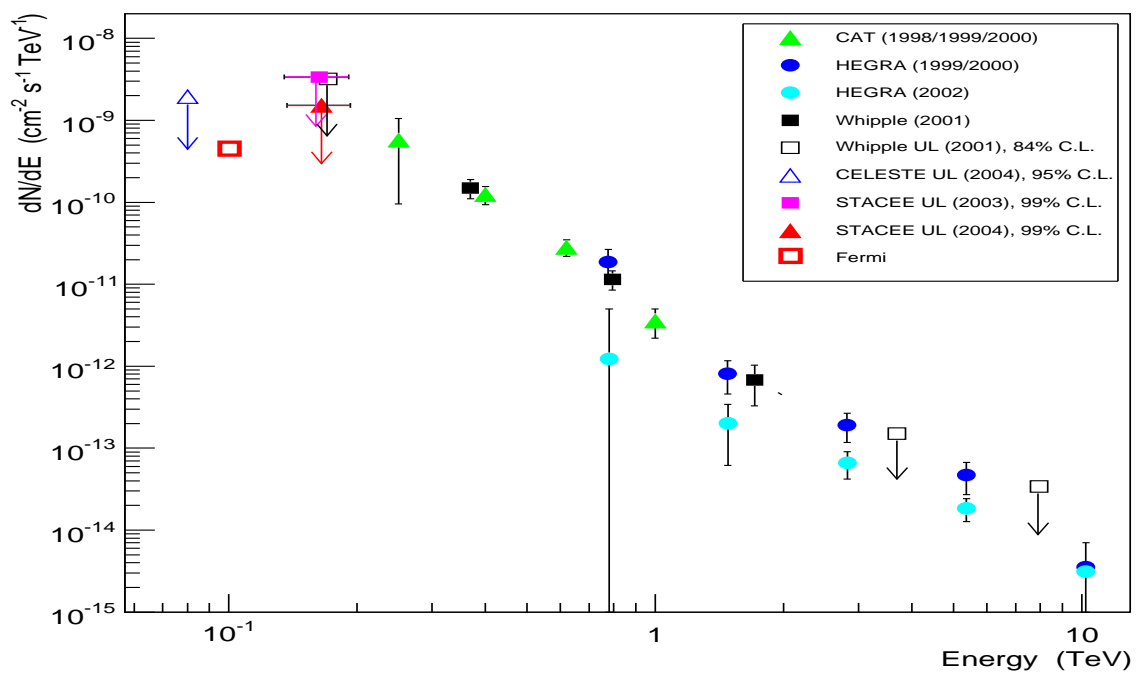

Figure 3. Gamma-ray spectrum of H 1426+428 with the STACEE 99\% flux upper limits at $164 \mathrm{GeV}$. Most other measurements and upper limits are from imaging Cherenkov telescopes. The Fermi point is an extrapolation from lower energy data to $100 \mathrm{GeV}$. Note that the measurements are not contemporaneous.

As was done for the 1ES 1218+304 data, detailed Monte Carlo simulations of the detector were used to calculate the effective area. The simulations were done separately for the two canting schemes and for several detector pointing directions (hour-angles). Weighting according to the hour-angle distribution of the data then results in an overall effective area function (for each data set); these curves are similar to those of Figure 1 .

Each effective area curve is then folded with a power law spectrum to generate the detector response function. For $\mathrm{H}$ 1426, we assume a spectral index of -3.50 ([ [22], [ 23],[25]). The resulting STACEE response function peaks at $164 \mathrm{GeV}$; the estimated systematic uncertainty on this value is $28 \mathrm{GeV}$.

The detector response functions and the measured rate of excess events are then used to derive $99 \%$ CL flux upper limits at $\mathrm{E}_{t h}$ of $164 \mathrm{GeV}$ of $3.4 \times 10^{-9} \mathrm{~cm}^{-2} \mathrm{~s}^{-1} \mathrm{TeV}^{-1}$ and $1.5 \times 10^{-9} \mathrm{~cm}^{-2} \mathrm{~s}^{-1} \mathrm{TeV}^{-1}$ for the 2003 and 2004 data sets, respectively. These results are shown in Figure 3 together with measurements at higher energies by imaging Cherenkov telescopes, and an extrapolation of lower energy Fermi data to our energy range (again using the measured Fermi spectral index and no EBL model).

\section{Conclusions and Summary}

Both 1ES $1218+304$ and $\mathrm{H} 1426+428$ are X-ray bright HBLs, and were promising targets for STACEE based on their broad-band SEDs. The main motivation for the STACEE observations was to obtain data on these two blazars in a largely-unexplored 
energy range. Together with observations of $\mathrm{H} 1426+428$ made with the CELESTE instrument [30], the data presented in this paper represent the only observations of these blazars below $200 \mathrm{GeV}$ by solar heliostat arrays.

Both sources have now been detected using Fermi's LAT instrument, with hard spectra [7]. Indeed, their spectra rank as among the hardest of the $38 \mathrm{TeV}$-selected AGN that Fermi has observed, reaffirming the interest of having observational data in the STACEE energy range.

In the case of $1 \mathrm{ES} 1218+304$, the extrapolation of the measured Fermi GeV spectrum to the STACEE threshold energy $E_{t h}$ results in a differential flux that is less than a factor of two below the upper limit reported here. Our upper limit is also comparable to the measured MAGIC flux in this energy range, and to extrapolations of higher-energy observations by VERITAS (see Figure 2).

In the case of $\mathrm{H} 1426+428$, the Fermi GeV spectrum is nearly an order of magnitude below our upper limit reported here. However, our limit is comparable to the extrapolation from higher energies of imaging telescope data (see Figure 3). This again points out the importance of data in the energy range of the solar-array telescopes like STACEE - this is the range where the GeV spectrum (Fermi measures a spectral index of $\Gamma \approx 1.5[7]$ ) transitions to the TeV spectrum (with a spectral index of 3.5).

Many AGNs are known to be highly variable sources in the VHE regime. For example, VERITAS recently observed a flare from 1ES 1218+304 with an estimated flux-doubling time of one day [35]. For both sources reported here, the STACEE data was accumulated over several years, and the upper limits represent mean flux levels over the observational period. The energy range reported on here is worthy of investigation by higher sensitivity instruments such as a new generation of imaging arrays.

In summary, we have presented data from STACEE observations of two HBL candidates suggested as potential TeV emitters by Costamante and Ghisellini [ 17], 1ES 1218+304

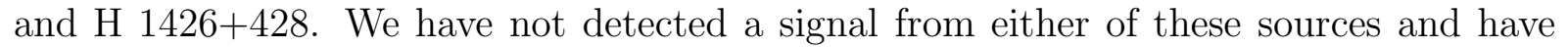
set upper limits on their gamma-ray flux levels, at an energy below that of most of the imaging atmospheric Cherenkov telescopes detections.

\section{Acknowledgements}

We are grateful to the staff at the National Thermal Solar Test Facility for their enthusiastic and professional support. This work was funded in part by the U.S. National Science Foundation, the University of California, Los Angeles, the Natural Sciences and Engineering Research Council, le Fonds Québecois de la Recherche sur la Nature et les Technologies, the Research Corporation, and the California Space Institute.

\section{REFERENCES}

1. J. A. Hinton and W. Hofmann, Teraelectronvolt Astronomy, Ann. Rev. Astron. Astrop. 47, 523, 2009

2. C. M. Urry and P. Padovani, PASP, 107, 715 (1995)

3. M. Böttcher, Astrophys. SpaceSci. 309, 95-104 (2007)

4. A. Mücke, R. J. Protheroe, R. Engel et al., Astropart. Phys. 18, 593 (2003) 
5. G. Fossati et al., MNRAS, 299, 433 (1998)

6. R. C. Hartman et al., ApJS, 123, 79 (1999)

7. A. A. Abdo et al., ApJ, 707, 1310 (2009)

8. A. A. Abdo et al., ApJ, 715, 429 (2010)

9. J. Albert et al., ApJL, 642, 119 (2006)

10. V. A. Acciari et al., ApJL, 684, L73 (2008)

11. V. A. Acciari et al., ApJL, 693, L104 (2009)

12. V. A. Acciari et al., ApJL, 708, L100 (2009)

13. T. Lindner et al., APh, 28, 388 (2007)

14. D.A. Bramel et al., ApJ, 629, 108 (2005)

15. L. M. Boone et al., ApJ, 579, L5 (2002)

16. J. E. Carson et al., ApJ, 662, 199 (2007)

17. L. Costamante and G. Ghisellini, A\&A, 384, 56 (2002)

18. K. Mannheim, A\&A, 269, 67 (1993)

19. L. Costamante et al., A\&A, 371, 512 (2001)

20. R. Sato et al., ApJ, 680, L9 (2008)

21. V. A. Acciari et al., ApJ, 695, 1370 (2009)

22. D. Horan et al., ApJ, 571, 753 (2002)

23. D. Petry et al., ApJ, 580, 104 (2002)

24. F. Aharonian, A\&A, 384L, 23 (2002)

25. A. Djannati-Atai, A\&A, 391L, 25 (2002)

26. D.M. Gingrich et al., IEEE Transactions on Nuclear Science, 52, 2977 (2005)

27. J. P. Martin \& K. Ragan, IEEE NSS/MIC Conf., Lyon, France, Vol. 2, 141 (2000)

28. D.S. Hanna et al., Nucl. Instrum. and Meth. A489, 126 (2002)

29. R.A. Scalzo et al., ApJ, 607, 778 (2004)

30. D.A. Smith et al., A\&A 459, 453 (2006)

31. J. Kildea et al., Proc 29th Int. Cosmic Ray Conference (Pune) 5: 135 (2005)

32. J. Kildea et al., Proc 29th Int. Cosmic Ray Conference (Pune) 4: 89 (2005)

33. R. Mukherjee et al., Proc 30th Int. Cosmic Ray Conference (Merida) 3: page 925 (2008); arXiv:0710.4170

34. T. Li and Y. Ma, ApJ 272, 317 (1983)

35. V. A. Acciari et al., ApJ, 709, L163 (2010) 